\title{
Effectiveness and Usability of a Developed Collaborative Online Tool for Children with ADHD
}

\author{
Doaa Sinnari $^{1(\bowtie)}\left(\mathbb{D}\right.$, Paul Krause ${ }^{1}$, and Maysoon Abulkhair ${ }^{2}$ \\ 1 Faculty of Engineering and Physical Sciences, \\ University of Surrey, Guildford, UK \\ \{d. sinnari, p.krause\}@surrey.ac.uk \\ ${ }^{2}$ Information Technology Department, King Abdulaziz University (KAU), \\ Jeddah, Kingdom of Saudi Arabia \\ mabualkhair@kau.edu.sa
}

\begin{abstract}
This study evaluated the effectiveness and usability of a developed collaborative online tool (chit-chat) for children with Attention Deficit Hyperactivity Disorder (ADHD). We studied whether this tool influenced children's Knowledge and experience exchange, motivation, behavioral abilities and social skills while using another learning tool, ACTIVATE. A total of seven Saudi children with ADHD aged from 6 to 8 years were assigned to the collaborative intervention using iPads. They were asked to play mini games that positively affect children with ADHD cognitively and behaviorally, then chat using our developed collaborative online tool, for three sessions. Progress points were measured and quantitatively analyzed before and after the intervention, thematic analysis was applied on the qualitative data. Participants showed improvements in overall performance when using the learning tool ACTIVATE. Ecollaboration was found to be effective to children with ADHD and positively influencing their knowledge, experience, motivation and social skills.
\end{abstract}

Keywords: ADHD $\cdot$ E-collaboration $\cdot$ Effectiveness $\cdot$ Usability

\section{Introduction}

There is a lack of research on the effectiveness of using online collaborative closedcommunities designed especially for children with ADHD that might have an effect on their abilities, skills, and performance in educational activities. Moreover, in our previous work, we investigated an e-game developed to enhance abilities and skills of children with ADHD, and positive results were reported (Sinnari et al. 2018). Yet, one of the important issues that arose was not having any kind of live interaction or collaboration between the children who played with it, as each child played individually. According to the reviewed literature, collaboration is one of the most effective learning strategies. It reinforces motivation and knowledge exchange and builds social skills (Huang et al. 2017). Children learn by communicating, interacting, and imitating. By letting them work closely together, they will experience high levels of engagement, motivation, and enjoyment (Xie et al. 2008). Thus, to add a missing component to the 
evaluated e-game, we developed and evaluated a collaboration tool, Chit-Chat, as an add-on for user engagement. The overview of Chit-Chat, the research methodology, and the results are reported in this study.

\subsection{Research Questions}

This experimental study investigated the following question:

- How does the use of the collaboration tool by children with ADHD in school settings affect their motivation, knowledge and experience and social behavior towards e-learning activities and environment?

- Is the developed collaboration tool interface usable and subjectively pleasing for children with ADHD?

\section{Related Work}

Evidence showed the optimistic impact of applications that support collaborative work among students on their motivation to learn, engagement with others, knowledge sharing, social skills and problem solving (Järvelä et al. 2015). Therefore, many researchers, developers, and healthcare specialist were encouraged to extend their work, during last decade, by developing special applications and serious games that target and promote some of healthcare students' weaknesses. But still few of them explored the impact of e-collaboration and actually integrated this strategy in their studies. There was a study found on the effect of collaborative intervention using different tools for children with dyslexia (Vasalou et al. 2017) which found emphasizing their social engagement; another one was explored with autistic children (Holt and Yuill 2017), in which they used double-iPad approach to encourage and facilitate communication with peers and adults. For younger children, a study found a promising benefit of a collaborative experience for kindergarten children with Learning difficulties (Drigas et al. 2015), this strategy helped them learn simple math in a fun way. Another study, targeted autistic children, developed a collaborative game that encouraged social interaction and cognitive abilities (Barajas et al. 2017). There was only one study, to my knowledge, that investigated the effectiveness of a collaborative serious game "plan it commander", on children with ADHD (Bul et al. 2015). The game included three mini games that offer neurocognitive and behavioural training tasks. Children collaborate with their parents only on some behavioural daily-life tasks such as: planning, time management, responsibilities, and problem solving. "Plan it commander" focused on resolving cognitive, behavioural, and social, as real-life problem-solving, issues.

\section{Chit-Chat Overview}

The Chit-Chat tool is a web-based application that offers a chatting panel, which is managed and monitored by teachers, for children with ADHD. The Chit-Chat interface was designed and developed to complement an e-game "ACTIVATE" (i.e. an add-on 
feature to help children interact between mini games). We used the 15 guidelines proposed by McKnight (2010) to design usable interfaces for children with ADHD. It contains two portals: one for teachers with more control and the other for children. Each user has to log in to the system using a unique user name and password (Fig. 1).
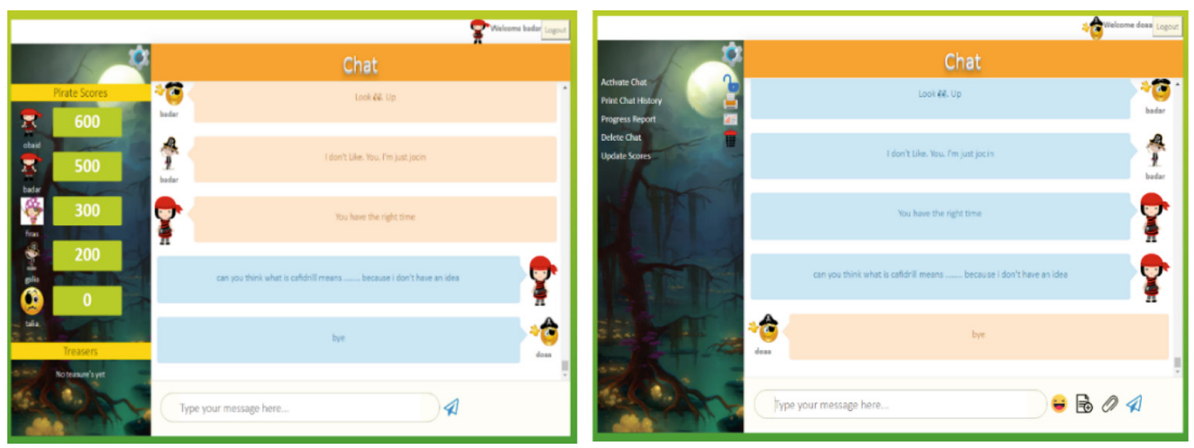

Fig. 1. Teacher portal with controls (right) and children portal (left).

In the student account, they can chat, send emojis, view their achieved progress scores in ACTIVATE, view their friends' progress scores, change their character avatar, and convert text to audio by tapping posted text/chat. The teacher account provides controls to activate or deactivate chat, clear/delete chat, change the character avatar, create multiple-choice challenges with timer options, print chat history, and generate progress reports for each child showing accuracy and speed averages in challenges.

\section{Experiment Design and Methodology}

One of the issues was the inability to develop a chatting feature within ACTIVATE itself due to a limitation from the developing company. Therefore, we decided to build an add-on application. The idea was running ACTIVATE games synchronously with the Chit-Chat application to make the students feel that they were using a single application.

Relying on our research objectives, we designed a qualitative and quantitative experimental study. The independent variable was the e-collaborative activity tool (the Chit-Chat application), and the dependent variables were all the following behaviors, which were measured and assessed in the experiment: Motivation to complete tasks, socializing, knowledge, and experience.

The experiment included three mini interventions. A pilot study was done three weeks prior to the actual experiment to test the study design to understand the time needed for each session and to test the wording of the tasks. A usability test was done during the intervention to study the interface design and discover more usability issues. 
Before we started the intervention experiment, a baseline session was prepared to allow participants play with ACTIVATE alone for $20 \mathrm{~min}$. Then, the intervention sessions were held on three days for $35 \mathrm{~min}$ each in total: $20 \mathrm{~min}$ for ACTIVATE and 15 min for the Chit-Chat application.

ACTIVATE contains four mini games per session. Each game takes only five minutes to complete. Thus, our idea was to slot in the Chit-Chat application for five minutes after each ACTIVATE mini game. There will be three sub-sessions of ChitChat in a single intervention. Children used the iPad as the tool of study.

In the first chatting sub-session, participants were asked to do certain usability tasks. In the second sub-session, the teacher initiated the chat by introducing himself and asked the participants to do so and to talk about themselves a little bit. Using this activity, the researcher studied the effect of a collaborative activity on the children's socialization. In the third sub-session, after playing the third ACTIVATE mini game, the teacher asked about the ACTIVATE games. The teacher asked: What is the best game? How can one get a higher score? What are some secret hints to share with other participants? We wanted to know to what extent the collaborative activities encourage children to exchange their knowledge and experience about given tasks.

In the second intervention, the teacher revealed the game scores for all the participants on the chatting panel. The chatting remained locked, and the children were given the chance to see their scores and their friends' scores for about one minute. We wanted to investigate the effect of seeing their peers' scores regarding whether it would motivate them to do better or worse in the next tasks.

In our last intervention, the researcher used the same experimental design for the first intervention. The only difference was that the teacher did not interfere in all three sub-session chats. We wanted to investigate whether the teacher's presence and participation affected this collaborative activity positively or negatively.

\section{Participants}

Seven children, all diagnosed with ADHD (three females and four males), were recruited to participate in this study. The students were from Grades 1, 2, and 3 from Al-Nojood International Private School. Pre-intervention demographic questionnaire and Conner rating were used to identify the type of ADHD, level of severity, and social skills for the participants, which were filled in by parents (Table 1). They were all familiar with the iPad and used it regularly in playing online games and viewing YouTube clips. However, the students had never used any chatting applications before.

\section{Data Collection and Analysis}

There were two types of data collected: quantitative and qualitative. The quantitative part was provided by ACTIVATE system, which measures each participant's overall progress in mini games against their peers. We used the baseline and the postintervention progress measures for each participant to check their performance before 
and after the intervention. To statistically analyse their progress points, we used the paired t-test to evaluate the significance by calculating the p-value.

For the qualitative part, some of the participants' behavioural responses were recorded (written) by the researcher while interacting with the application, such as body and hand movements and interacting with each other. Video recording was prohibited due to school policy, but we had approval from the head of the school to take pictures without showing participants' faces. Moreover, we managed to get approval to record audio tracks of post-intervention interviews with the participants to capture their experience with the collaboration tool.

Table 1. Participant demographic data, ADHD type, and sociability

\begin{tabular}{l|l|l|l|l|l}
\hline Participants & Age (years) & ADHD type & Academic level & On treatment? & Sociability? \\
\hline Bader & 7 & Combined & Medium & No & Limited friends \\
\hline Firas & 6 & Combined & Low & No & Social \\
\hline Galia & 6 & Hyperactive & Medium & Yes (medication) & Shy-no friends \\
\hline Obaid & 7 & Inattentive & Medium & No & Social \\
\hline Sarah & 7 & Inattentive & Low & No & Shy-few friends \\
\hline Suhaib & 8 & Combined & Low & No & Shy-few friends \\
\hline Talia & 8 & Inattentive & Medium & No & Limited friends \\
\hline
\end{tabular}

Thematic analysis (Clarke and Braun 2014) was applied to the qualitative data gathered from participants' chat history, observational notes, and audio recordings of post-intervention open questions. This process allowed us to interpret the gathered raw data and present it in a more intensive construction of themes. To test usability and satisfaction, a usability test was done in the first session of the intervention. For the user experience, an open-ended interview was used. These feedback items were included in the thematic analysis as part of our gathered data.

\section{Usability Test}

In designing the Chit-Chat interface, we used some of the guidelines suggested from literature for children with ADHD and applied usability guidelines (McKnight 2010). The main idea was to keep it as simple and easy as possible and less distracting. Therefore, we asked the participants and a teacher to do the usability tasks on the ChitChat portal. As Nielsen (2000) stated, a usability test carried out by only five participants will disclose $85 \%$ of the usability issues.

The teacher completed all the tasks with no difficulties. However, some students raised issues, and we dealt with them accordantly. 


\section{Findings and Discussion}

\subsection{Effectiveness of the Collaboration Tool}

For our quantitative data, we measured the effectiveness of our tool by comparing the participants' performance before and after the intervention. The statistical significance was determined by a paired t-test using GraphPad PRISM (v. 5.0, GraphPad Software, Inc.). A p-value of less than 0.05 was considered significant.

Table 2 shows the progress points scored by each participant before and after the intervention. Each progress point is a measure of the levels completed and the speed within the e-game. The distribution of the progress points differences was fairly normal. As our baseline measures, the pre-intervention mean was $\mathrm{M}=15.57$, with a standard deviation $\mathrm{SD}=2.30$. After intervention, they were $\mathrm{M}=21.57$, and $\mathrm{SD}=1.27$.

Table 2. Progress points by participants before and after the intervention.

\begin{tabular}{l|l|l|l|l|l}
\hline \multirow{2}{*}{ Participants } & \multicolumn{3}{l|}{ Progress points per session } & \multicolumn{2}{l}{$\begin{array}{l}\text { Percentage of } \\
\text { improvement }\end{array}$} \\
\cline { 2 - 6 } & Baseline & \multicolumn{2}{l}{ Post-intervention } & \multicolumn{1}{l}{ in } \\
\hline Bader & 17 & 18 & 37 & 58 & $12 \%$ \\
\hline Firas & 12 & 11 & 26 & 47 & $25 \%$ \\
\hline Galia & 14 & 20 & 42 & 63 & $57 \%$ \\
\hline Obaid & 16 & 14 & 33 & 55 & $19 \%$ \\
\hline Sarah & 14 & 15 & 31 & 51 & $14 \%$ \\
\hline Suhaib & 18 & 18 & 42 & 64 & $41 \%$ \\
\hline Talia & 18 & 20 & 41 & 65 & $17 \%$ \\
\hline
\end{tabular}

The two-tailed $\mathrm{p}$-value was less than 0.0001 ( $\mathrm{p} \leq 0.05$ ); thus, we rejected the null hypothesis. By conventional criteria, this difference was statistically significant. The mean of the baseline minus the post-intervention is -6.00 (95\% confidence interval (CI) $[-7.60,-4.40])$. As a conclusion, participants' performance in ACTIVATE was positively affected by applying the online collaboration tool (Chit-Chat; $t(6)=9.165$, $\mathrm{p} \leq 0.05)$.

There was some stability in the performance between the baseline and the first intervention session; participants have earned nearly the same amount of points. However, in the second session, we spotted a progressive increase in performance. By computing the difference in performance between the first and second sessions of intervention, we found an improvement in performance at an average of $26 \%$.

Theoretically, one of the reasons was due to motivating them by displaying participants' scores for all of them on the collaboration tool. Regarding the literature, many studies encourage posting scores for all students, so that each student can be positively motivated by others' scores (Klingberg et al. 2005; Ali and Puthusserypady 2015). Another reason is that participants in the first session exchanged some of the playing techniques and hints that helped them personally increase their scores, such as speed, concentrating on the monkey with fingers close to the screen, easy games versus 
hard games, and making fewer mistakes the higher the mouse goes up. By the second session, some of them were actually applying these techniques while they were playing. The observational notes and chat history demonstrate the positive effects of exchanging knowledge and experience on improving performance. This was in line with the findings of a study (Fiers 2017) that utilized peer tutoring and information exchange among students with emotional and behavioral disorders. It showed growth in cognitive skills and gains in problem-solving proficiency. As motivation and knowledge exchange were dependent variables in our study, more details and qualitative results are reviewed in the next section.

\subsection{Thematic Analysis Results}

In the second part, our qualitative results were built upon the thematic analysis. All the transcribed information was translated from Arabic to simple English, considering the same simple expression level. Next, will discuss the most important and prominent codes.

\section{Knowledge and Experience Exchange}

At the opening five minutes, participants $(n=3)$ were very shy to contribute in the chat, they told the researcher that they do not know how to spell the words correctly. The researcher expressed to them that there was no need to worry about perfectly spelled words; just write it in any way and try your best. Participants were encouraged one by one to chat $(n=4)$. It was clear that the participants were able to use the chatting panel in exchanging what they knew and learned.

Our findings were in contrast with an earlier study that found negative effects of an online collaborative experience on children with behavioral disorders (Lipponen et al. 2003). Our findings showed positive conversations, socialization, and information exchange. Another study explained that low progress students struggle to ask for help (Kroesbergen et al. 2004). Again, in our study, a good number of help requests were raised from some of the low progress participants.

Yet, many studies were in line with our findings (Rief 2016). It has been shown that the peer-tutoring approach has promising effects on children with learning and behavioral disorders (DuPaul and Stoner 2014). Another finding confirmed exchanging information stimulates students' abilities (Tsuei 2014). Moreover, Vasalou et al. (2017) evaluated the experience of students with learning difficulties in exchanging knowledge, which resulted in improvements on their academic achievements.

Returning to our second finding, we noticed another interesting aspect; participants were giving positive feedback to each other $(n=4)$. These kinds of reactions would increase their self-esteem and confidence and encourage others to improve their performance (Van Popta et al. 2017). A different form of feedback was observed - a sort of a light and respectful criticism was introduced.

Our outcome was in line with a study that specified that children with ADHD are affected by positive feedback yet are less considerate of negative feedback (Bul et al. 2015). Another study found that written peer-interaction with supportive talk increases learning and improves social skills (Genlott and Grönlund 2016). 


\section{Motivational Influence}

This theme was mainly detected by the observed behavior and reactions of the participants. The second intervention was designed specifically to post game scores attained by all participants. Participants were able to see their scores and their peers' scores. Few studies have emphasized exposing group scores to reinforce motivation and to improve performance (Klingberg et al. 2005; Ali and Puthusserypady 2015). Participants $(n=6)$ showed excitement by moving their hands quickly up and down, jumping, and saying 'yes' or 'yay!!'. Another sign of motivation was noticed by the researcher; after score posting, some participants $(n=3)$ were taking a bit more time thinking about the task, concentrating, and progressing well. In addition, two participants $(n=2)$ shared how many reward stars they received.

Another factor that helped the researcher validate motivation was the level of engagement and activity in the chatting tool. To motivate the children's engagement in any game with others, factors such as challenge, competition, and interaction must be applied (Yee 2006). To my knowledge, there was no study found on the relation of the engagement level with the motivation for children with ADHD while using collaboration tools. Yet, we found one study (Ronimus et al. 2014) that showed that there is no significant effect on children's engagement by challenge level or reward system. From our point of view, the factors of peer interaction and competition were not presented in their work.

\section{Socialization}

One of the main symptoms of children with ADHD is having some difficulties in their social skills and interaction with peers (Wilkes-Gillan et al. 2017). Our objective in this study was to help them develop these skills by engaging them in a close collaborative online community. This assisted in reducing the fear of confrontation and encouraged them to release their feelings and opinions about certain games. In the first session, a few participants $(n=3)$ talked about their classes, favorite subjects, and things they like to eat. Others $(n=2)$ were too shy to write anything. They were observers rather than participators. In fact, they were only posting emojis as a way of interacting, but they were eventually encouraged by feedback from their peers who commented and interacted with their input. By the third intervention, some participants $(n=2)$ were sharing short jokes, and others $(n=2)$ were planning to play in the playground after the intervention. From the observation, we found that this experience has reflected positively on their prosocial relation outside the playing sessions. The concepts of indirect learning, emotional feedback, and facilitating mastery of given tasks, which are the fundamental elements of behavior development in the social cognitive theory (Bandura 1989), were executed in the intervention design.

A study done by McHale (2010) revealed that children with ADHD normally encounter difficulties in public online social communities owing to lack of safety and differences in reaction speed, cognitive abilities, and social skills. Children with this disorder usually are segregated and downgraded in social life; therefore, providing them a safe monitored online community will positively support them. Another study that was in line with our findings investigated an online communication system designed for children with ADHD. They found that children are more confident, open 
to self-identity, and seek support if needed from their peers more easily online than in real-life situations (Raskind et al. 2006).

\subsection{Chit-Chat Usability and Satisfaction}

One of our essential goals in this study was to evaluate the Chit-Chat interface in terms of usability and satisfaction. As we stated earlier, a usability test was done on the first session of the intervention with the same group of participants $(n=7)$. They were given certain tasks to perform, and they were encouraged to talk aloud during the test. Detected usability issues were fixed on the same day, and a post-intervention openended interview was done to assess their experience with the tool.

Nielsen (1994) identified usability using five quality components that must be applied, to any system in which humans might interact. Thus, any system interface would be considered usable if it was efficient, learnable, memorable, and satisfactory and has a small error rate.

The quantitative results showed that Chit-Chat is an effective collaboration tool for children with ADHD. We found fairly significant improvement in the participants' performance after comparing their achievements in ACTIVATE before and after using the tool. From these findings, the Chit-Chat collaboration tool is efficient.

The remaining quality components were measured by seeking the participants' feedback and experience with the tool, combined with our observational notes while they were using the application. A post-intervention interview was conducted with each participant individually. We did not use the questionnaire type due to their young age and probable struggle of reading, comprehending, and writing well-constructed and explanatory sentences. Therefore, we used interview-like questions with the aid of five printed smiley's that ranged from strongly agree to strongly disagree to help them show their emotions about a specific question, and then talk about the 'why?' afterwards.

The interview took approximately ten minutes with each participant. They were asked multiple questions that relate to each quality component. All participants $(n=4)$ strongly agreed and $(n=3)$ agreed that the system was simple and easy to use from the first time they interacted with the interface. Two participants $(n=2)$ found that the chat deactivation feature was confusing in the beginning, but in less than a minute they noticed the statement 'the chat is locked by the teacher' and immediately understood and waited until the teacher unlocked the chat. Regarding the avatar icon, and after changing it from a gear (hat resembles settings to a small human character (s), they easily knew how to change their avatar character. The participants did not face any problems with converting text to audio. Most of them $(n=5)$ tapped the text directly when we asked them to hear what they wrote on usability test and recognized the lefthand side when they were asked about scores. From these user experiences with the tool, Chit-Chat is learnable.

On the third intervention, all the participants $(n=7)$ remembered how to reach all the features within the Chit-Chat interface. They were using the tool with confidence and skill. The features were few, easy to reach, and all on the same page; therefore, the Chit-Chat interface is memorable.

As expected, due to its nature as a chat-only tool, the error rate was nearly none. There was no wrong way of doing something. All the participants completed the tasks 
they were asked to do correctly and chatted through the panel without mistakes. They knew the use of each icon, and no multi-steps nor multi-levelled tasks were required. For that reason, the Chit-Chat interface has no error rate.

The design was kept simple and easy with less distraction. All participants $(n=7)$ strongly agreed that they liked the theme, colors, and icons in the interface. A few of them $(n=2)$ suggested that they want to customize their pirate avatars and dress them up, and one $(n=1)$ asked about whether they could have more control of the text in terms of changing the color, font, and size. All these suggestions will be taken in consideration when modifying the tool in the future for another evaluation. Therefore, the participants agreed that Chit-Chat was subjectively pleasing, and they were satisfied with the overall interface design.

From these previous findings and feedback, Chit-Chat is efficient, learnable, memorable, and satisfactory with no error rate. Thus, the Chit-Chat collaboration tool is considered usable and satisfactory.

\section{Conclusion}

The outcomes of the current study fit into the forthcoming projects of online collaborative interventions for children with ADHD. The online tool Chit-Chat was designed and developed to validate the 'e-collaboration' concept through providing a chatting panel to engage participants. The intervention took place in an international primary school in Jeddah, Saudi Arabia. Seven students with ADHD aged between 6 and 8 years participated in the study. The intervention involved three mini interventions, each with four ACTIVATE mini games, and a chatting session after each game. Children showed fairly significant improvements in their performance while playing ACTIVATE. Chit-Chat, the online collaboration tool, was found to positively influence children's knowledge and experience exchange, motivation, and social skills. In addition, the Chit-Chat tool was effective, usable, and subjectively pleasing. The results did not reveal improvements in the following participants' cognitive abilities: attention, processing speed, and working memory. This could be clarified by the fact that the developed collaboration tool did not aim to target these types of abilities by itself. It was developed to be integrated with another system, ACTIVATE, that works on improving those abilities. No direct nor noticeable effects on the children's behavior were found. As for future work, it is recommended to repeat the evaluation with a larger sample size and longer duration, perhaps including a control group to explore how does diversity affects the interaction with the tool.

\section{References}

Ali, A., Puthusserypady, S.: A 3D learning playground for potential attention training in ADHD: a brain computer interface approach. In: 2015 37th Annual International Conference of the IEEE Engineering in Medicine and Biology Society (EMBC), pp. 67-70. IEEE, August 2015 Bandura, A.: Human agency in social cognitive theory. Am. Psychol. 44(9), 1175 (1989) 
Barajas, A.O., Al Osman, H., Shirmohammadi, S.: A serious game for children with autism spectrum disorder as a tool for play therapy. In: 2017 IEEE 5th International Conference on Serious Games and Applications for Health (SeGAH), pp. 1-7. IEEE, April 2017

Bul, K.C., et al.: Development and user satisfaction of "Plan-It Commander", a serious game for children with ADHD. Games Health J. 4(6), 502-512 (2015)

Clarke, V., Braun, V.: Thematic analysis. In: Michalos, A.C. (ed.) Encyclopedia of Critical Psychology, pp. 1947-1952. Springer, New York (2014). https://doi.org/10.1007/978-94007-0753-5

Drigas, A., Kokkalia, G., Lytras, M.D.: ICT and collaborative co-learning in preschool children who face memory difficulties. Comput. Hum. Behav. 51, 645-651 (2015)

DuPaul, G.J., Stoner, G.: ADHD in the Schools: Assessment and Intervention Strategies. Guilford Publications, New York (2014)

Fiers, J.: The effects of peer tutoring on math fact fluency of elementary students with emotional and behavioral disorders. Doctoral dissertation, Western Illinois University (2017)

Genlott, A.A., Grönlund, Å.: Closing the gaps - improving literacy and mathematics by ICTenhanced collaboration. Comput. Educ. 99, 68-80 (2016)

Holt, S., Yuill, N.: Tablets for two: how dual tablets can facilitate other-awareness and communication in learning disabled children with autism. Int. J. Child-Comput. Interact. 11, 72-82 (2017)

Huang, C.S., Su, A.Y., Yang, S.J., Liou, H.H.: A collaborative digital pen learning approach to improving students' learning achievement and motivation in mathematics courses. Comput. Educ. 107, 31-44 (2017)

Järvelä, S., et al.: Enhancing socially shared regulation in collaborative learning groups: designing for CSCL regulation tools. Educ. Technol. Res. Dev. 63(1), 125-142 (2015)

Klingberg, T., et al.: Computerized training of working memory in children with ADHD-a randomized, controlled trial. J. Am. Acad. Child Adolesc. Psychiatry 44(2), 177-186 (2005)

Kroesbergen, E.H., Van Luit, J.E., Maas, C.J.: Effectiveness of explicit and constructivist mathematics instruction for low-achieving students in the Netherlands. Elementary Sch. J. 104(3), 233-251 (2004)

Lipponen, L., Rahikainen, M., Lallimo, J., Hakkarainen, K.: Patterns of participation and discourse in elementary students' computer-support collaborative learning. Learn. Instr. 13, 487-509 (2003). https://doi.org/10.1016/s0959-4752(02)00042-7

McHale, T.J.: Delayed maturation of neuropsychological abilities in ADHD: a developmental comparison between children, adolescents, and adults. Doctoral dissertation (UMI No. 3449435), Fielding Graduate University, Santa Barbara, CA (2010)

McKnight, L.: Designing for ADHD in search of guidelines. In: IDC 2010 Digital Technologies and Marginalized Youth Workshop (2010)

Nielsen, J.: Why You Only Need to Test with 5 Users. NNGroup, Nielsen Norman Group, USA (2000). https://www.nngroup.com/articles/why-you-only-need-to-test-with-5-users/. Accessed 02 Jan 2017

Nielsen, J.: Usability Engineering. Elsevier, Amsterdam (1994)

Raskind, M., Margalit, M., Higgins, E.: "My LD": children's voices on the internet. Learn. Disabil. Q. 29(4), 253-268 (2006)

Rief, S.F.: How to Reach and Teach Children and Teens with ADD/ADHD. Wiley, Hoboken (2016)

Ronimus, M., Kujala, J., Tolvanen, A., Lyytinen, H.: Children's engagement during digital game-based learning of reading: the effects of time, rewards, and challenge. Comput. Educ. 71, 237-246 (2014) 
Sinnari, D., Krause, P., Abulkhair, M.: Effects of e-games on the development of Saudi Children with attention deficit hyperactivity disorder cognitively, behaviourally and socially: an experimental study. In: Antona, M., Stephanidis, C. (eds.) Universal Access in HumanComputer Interaction. Methods, Technologies, and Users. LNCS, vol. 10907, pp. 598-612. Springer, Cham (2018). https://doi.org/10.1007/978-3-319-92049-8_44

Tsuei, M.: Mathematics synchronous peer tutoring system for students with learning disabilities. J. Educ. Technol. Soc. 17(1), 115-127 (2014)

Van Popta, E., Kral, M., Camp, G., Martens, R.L., Simons, P.R.J.: Exploring the value of peer feedback in online learning for the provider. Educ. Res. Rev. 20, 24-34 (2017)

Vasalou, A., Khaled, R., Holmes, W., Gooch, D.: Digital games-based learning for children with dyslexia: a social constructivist perspective on engagement and learning during group gameplay. Comput. Educ. 114, 175-192 (2017)

Wilkes-Gillan, S., Cantrill, A., Cordier, R., Barnes, G., Hancock, N., Bundy, A.: The use of video-modelling as a method for improving the social play skills of children with attention deficit hyperactivity disorder (ADHD) and their playmates. Br. J. Occup. Ther. 80(4), 196207 (2017)

Xie, L., Antle, A.N., Motamedi, N.: Are tangibles more fun? Comparing children's enjoyment and engagement using physical, graphical and tangible user interfaces. In: Proceedings of the 2nd International Conference on Tangible and Embedded Interaction, pp. 191-198. ACM, February 2008

Yee, N.: Motivations for play in online games. Cyberpsychol. Behav. 9, 772-775 (2006) 